\title{
OPEN Intrarenal Doppler ultrasonography reflects hemodynamics and predicts prognosis in patients with heart failure
}

\author{
Akiomi Yoshihisa ${ }^{1 凶}$, Koichiro Watanabe ${ }^{1}$, Yu Sato $^{1}$, Shinji Ishibashi $^{2}$, Mitsuko Matsuda ${ }^{2}$, \\ Yukio Yamadera ${ }^{2}$, Yasuhiro Ichijo ${ }^{1}$, Tetsuro Yokokawa ${ }^{1}$, Tomofumi Misaka ${ }^{1}$, \\ Masayoshi Oikawa ${ }^{1}$, Atsushi Kobayashi ${ }^{1}$ \& Yasuchika Takeishi ${ }^{1}$
}

We aimed to clarify clinical implications of intrarenal hemodynamics assessed by intrarenal Doppler ultrasonography (IRD) and their prognostic impacts in heart failure (HF). We performed a prospective observational study, and examined IRD and measured interlobar renal artery velocity time integral (VTI) and intrarenal venous flow (IRVF) patterns (monophasic or non-monophasic pattern) to assess intrarenal hypoperfusion and congestion in HF patients $(n=341)$. Seven patients were excluded in VTI analysis due to unclear imaging. The patients were divided into groups based on (A) VTI: high VTI (VTI $\geq 14.0 \mathrm{~cm}, \mathrm{n}=231$ ) or low VTI (VTI $<14.0 \mathrm{~cm}, \mathrm{n}=103)$; and (B) IRVF patterns: monophasic $(\mathrm{n}=36)$ or non-monophasic $(n=305)$. We compared post-discharge cardiac event rate between the groups, and right-heart catheterization was performed in 166 patients. Cardiac index was lower in low VTI than in high VTI $(P=0.04)$, and right atrial pressure was higher in monophasic than in non-monophasic $(P=0.03)$. In the Kaplan-Meier analysis, cardiac event rate was higher in low VTI and monophasic groups $(P<0.01$, respectively). In the Cox proportional hazard analysis, the combination of low VTI and a monophasic IRVF pattern was a predictor of cardiac events $(P<0.01)$. IRD imaging might be associated with cardiac output and right atrial pressure, and prognosis.

Heart failure (HF) is a widespread and serious problem that has been reported in many countries ${ }^{1-3}$, and causes multiple organ dysfunction through a combination of reduced arterial perfusion and passive congestion. The abdominal compartment, which contains the kidney, liver, splanchnic vasculature, gut, etc., has recently been focused upon in HF patients ${ }^{4-9}$. Central venous pressure (CVP) measured with right-heart catheterization (RHC) is a commonly used surrogate to evaluate organ congestion ${ }^{10,11}$. On the other hand, Nohria-Stevenson profiles demonstrated the clinical importance of assessments of perfusion ("cold" vs. "warm"), as well as congestion ("wet" vs. "dry") ${ }^{12}$. However, these profiles are relatively subjective.

Renal and cardiac functions have close and complementary interconnections; this is called the cardio-renal syndrome $(\mathrm{CRS})^{13}$. CRS is a disorder of the heart and kidneys whereby acute or chronic dysfunction in one organ may influence each other, and share common pathophysiology such as hemodynamics, neurohumoral, inflammatory, and oxidative injury ${ }^{14}$. Intrarenal congestion and hypoperfusion are critically involved in the pathophysiology of HF, and evaluation of renal function in HF patients is important ${ }^{15,16}$. Regarding renal congestion, it has recently been reported that intrarenal venous flow (IRVF) patterns (i.e. continuous, biphasic, or monophasic patterns) measured by intrarenal Doppler ultrasonography (IRD) are correlated with right atrial pressure (RAP), and a monophasic IRVF pattern was associated with higher RAP as well as worse outcome ${ }^{17}$. However, the usefulness of IRD to assess the clinical profiles, such as both hypoperfusion and congestion, has not been fully examined.

In the current study, we aimed to: (1) examine the intrarenal hemodynamics assessed by IRD (not only intrarenal congestion [IRVF patterns] but also intrarenal hypoperfusion [velocity time integral, VTI of interlobar renal artery]) in HF patients; and (2) clarify the clinical impacts of the IRD findings on prognosis in HF patients.

\footnotetext{
${ }^{1}$ Department of Cardiovascular Medicine, Fukushima Medical University, 1 Hikarigaoka, Fukushima 960-1295, Japan. ${ }^{2}$ Department of Clinical Laboratory Medicine, Fukushima Medical University, Fukushima, Japan. ${ }^{\boxplus}$ email: yoshihis@fmu.ac.jp
} 


\section{Results}

Comparisons of the high and low VTI groups are summarized in Table 1. The low VTI group was older, had a lower systolic blood pressure and higher heart rate, and had a higher prevalence of atrial fibrillation (AF), chronic kidney disease $(\mathrm{CKD})$, higher levels of $\mathrm{B}$-type natriuretic peptide (BNP), creatinine, urinary albumin creatinine ratio, and $N$-acetyl- $\beta$-D-glucosaminidase (NAG). In contrast, hemoglobin and estimated glomerular filtration rate (eGFR) were lower. The low VTI group also had higher levels of left atrial volume index (LAVI), early trans-mitral flow velocity to mitral annular velocity ratio (mitral valve E/e'), right atrial (RA) area, tricuspid regurgitation pressure gradient (TRPG), pulmonary artery pressure, RA pressure (RAP) and pulmonary artery wedge pressure (PAWP) and lower levels of left ventricular ejection fraction (LVEF), left ventricular outflow tract-VTI (LVOTVTI), right ventricular fractional area change (RV-FAC) and cardiac index. In addition, there were significant associations between interlobar renal VTI and age, heart rate, presence of New York Heart Association (NYHA) class III or IV, AF, hypertension, CKD, anemia, levels of hemoglobin, BNP, creatinine, eGFR, urinary albumin creatinine ratio, urinary NAG, LVEF, LAVI, LVOT-VTI, mitral valve E/e', RA area, RV area, RV-FAC, TRPG, pulmonary artery pressure, RAP and cardiac index. These results suggest that decreased VTI indicates intrarenal hypoperfusion, underlying low cardiac output, and leads to urinary albumin transudation or tubular damage.

Comparisons of the non-monophasic and monophasic groups are summarized in Table 2. The monophasic group were older, had a higher prevalence of AF, higher levels of BNP and lower levels of eGFR, higher levels of LAVI, mitral valve E/e', RA area, inferior vena cava diameter and TR-PG, and higher levels of RAP. In contrast, blood pressure, prevalence of hypertension, dyslipidemia, levels of creatinine, sodium, or cardiac index did not significantly differ between the groups. In addition, there were significant associations between monophasic IRVF pattern and age, presence of AF, levels of BNP, eGFR, LAVI, mitral valve E/e', RA area, inferior vena cava diameter, TRPG and RAP. These results suggest that a monophasic IRVF pattern indicates intrarenal congestion, and underlying increased CVP, RAP or right heart volume overload.

During the follow up period (mean $231 \pm 122$ days, range 12-523 days), 42 cardiac events including eight cardiac deaths and 34 worsening HF occurred. As shown in Fig. 1, in the Kaplan-Meier analysis, cardiac event rates were significantly higher in the low VTI group than in the high VTI group (log-rank $\mathrm{P}<0.01$ ), as well as in the monophasic group than in the non-monophasic group (log-rank $\mathrm{P}<0.01)$. In addition, as shown in Fig. 2, HF patients with low VTI and a monophasic IRVF pattern (subset 4) had the highest cardiac event rate (log-rank $\mathrm{P}<0.01$ ). In the univariate Cox proportional hazard analysis (Table 3), low VTI and a monophasic IRVF pattern were associated with a high cardiac event rate (low VTI, hazard ratio [HR] 3.65, 95\% CI 1.97-6.75; monophasic IRVF pattern, HR 2.86, 95\% CI 1.46-5.59), and the combination of low VTI and a monophasic IRVF pattern (vs. high VTI and non-monophasic patterns as reference) was a predictor of cardiac events in HF patients (HR 8.36, 95\% CI 3.37-20.75). These results suggest that the combination of interlobar renal VTI (intrarenal perfusion) and IRVF patterns (intrarenal congestion) might be a useful tool for evaluating renal hemodynamic subsets and estimating prognosis. With regard to the multivariate Cox proportional hazard analysis, as there were limited number of events ( 42 events) and multicollinearity between IRD findings and possible confounding factors such as parameters of demographics, comorbidities, laboratory data, echocardiography and right-heart catherization, we only adjusted for age and sex. Even after adjusting for age and sex, low VTI, a monophasic IRVF pattern and combinations of low VTI and a monophasic IRVF pattern still remain associated with high cardiac event rates.

Intrarenal congestion and hypoperfusion determined by IRD (IRVF patterns and interlobar renal artery VTI) are associated with adverse prognosis in HF patients.

\section{Discussion}

To the best of our knowledge, the present study was the first to report that (1) the associations between parameters of not only intrarenal congestion (IRVF patterns), but also of intrarenal hypoperfusion (interlobular renal artery VTI) determined by IRD and both RHC and echocardiography, and that (2) prognostic impacts of intrarenal congestion and hypoperfusion in patients with HF. The combination of interlobar renal VTI and IRVF patterns might be a useful tool for evaluating intrarenal hemodynamic subsets such as the Nohria-Stevenson profiles, and may be therapeutic targets.

Intrarenal congestion caused by increased CVP, namely increased right-sided pressure overload, has been one of the main pathophysiologic findings in CRS ${ }^{10,11,18-21}$. High CVP, rather than low cardiac output, predetermines the risk of worsening renal function in decompensated $\mathrm{HF}^{10}$. Previous studies have shown that IRVF patterns were associated with increased RAP levels, and correlated with clinical outcomes ${ }^{17,22}$. The increased CVP causes the increase of renal interstitial pressure and intrarenal parenchymal compliance around the intrarenal vessels, fibrosis, and leads the resistance of $\operatorname{IRVF}^{17,23,24}$. It has been reported that $22.5 \%$ of HF patients showed monophasic IRVF pattern ${ }^{17}$, and that the IRVF patterns could be changed depending on the renal congestion, and their changes were associated with renal impairment and poor prognosis ${ }^{25}$. In the present study, prevalence of monophasic IRVF pattern was $10.6 \%$, and was lower than $22.5 \%$ of previous study ${ }^{17}$. Although we could not fully explain the difference of their IRVF patterns, above changes in IRVF patterns ${ }^{25}$ depending on renal congestion might affect their differences. Increased renal interstitial pressure reduces hydrostatic and colloid osmotic pressure differences between glomerular capillaries and Bowman's space ${ }^{26}$. In addition, systemic venous congestion raises neurohormonal activation (e.g. renin-angiotensin system) resulting in renal vasoconstriction ${ }^{27,28}$. Neurohormonal activation reduces the $\mathrm{GFR}^{29,30}$, decreases plasma natriuretic peptide ${ }^{31}$, leads to HF progression, may contribute to multiple organ failure ${ }^{11,32}$, and results in adverse prognosis ${ }^{33}$.

Regarding intrarenal hypoperfusion, the results of the current study suggest that low interlobar renal artery VTI was associated with low cardiac index, low systolic blood pressure, and low eGFR. Hypotension leads to intrarenal hypoperfusion ${ }^{34}$. In pre-renal acute kidney injury, when renal hypoperfusion is sustained, eGFR is initially decreased without parenchymal damage ${ }^{35}$. The sustained inadequate oxygen and nutrient delivery to 


\begin{tabular}{|c|c|c|c|c|c|}
\hline & \multicolumn{3}{|l|}{ VTI group } & \multicolumn{2}{|l|}{ Correlation with VTI } \\
\hline & High VTI $(n=231)$ & Low VTI $(n=103)$ & P value & Correlation coefficient & P-value \\
\hline \multicolumn{6}{|l|}{ Demographics } \\
\hline Age (years) & $65.2 \pm 13.0$ & $69.6 \pm 12.3$ & $<0.01$ & -0.13 & 0.02 \\
\hline Male sex (n, \%) & $139(60.2)$ & $68(66.0)$ & 0.33 & -0.06 & 0.28 \\
\hline Body mass index $\left(\mathrm{kg} / \mathrm{m}^{2}\right)$ & $24.0 \pm 4.3$ & $23.3 \pm 5.3$ & 0.25 & 0.07 & 0.18 \\
\hline \begin{tabular}{|l|l|} 
Systolic blood pressure $(\mathrm{mmHg})$ \\
\end{tabular} & $120.1 \pm 17.1$ & $115.6 \pm 17.8$ & 0.03 & 0.11 & 0.05 \\
\hline Diastolic blood pressure $(\mathrm{mmHg})$ & $69.5 \pm 12.4$ & $68.4 \pm 12.9$ & 0.47 & -0.04 & 0.43 \\
\hline Heart rate (bpm) & $68.5 \pm 12.9$ & $75.5 \pm 15.0$ & $<0.01$ & -0.38 & $<0.01$ \\
\hline NYHA class III or IV (n, \%) & $11(4.8)$ & $11(10.7)$ & 0.06 & -0.12 & 0.03 \\
\hline \multicolumn{6}{|l|}{ Comorbidities } \\
\hline Atrial fibrillation (n, \%) & $71(30.7)$ & 49 (47.6) & $<0.01$ & -0.07 & $<0.01$ \\
\hline Hypertension (n, \%) & $134(58.0)$ & $71(68.6)$ & 0.07 & -0.05 & $<0.01$ \\
\hline Dyslipidemia (n, \%) & $163(70.6)$ & $75(72.8)$ & 0.70 & 0.04 & 0.42 \\
\hline Diabetes mellitus (n, \%) & $74(32.0)$ & $37(35.9)$ & 0.53 & -0.02 & 0.74 \\
\hline Chronic kidney disease (n, \%) & $99(42.9)$ & $74(71.8)$ & $<0.01$ & -0.30 & $<0.01$ \\
\hline Anemia (n, \%) & $88(38.1)$ & 57 (55.3) & $<0.01$ & -0.08 & $<0.01$ \\
\hline \multicolumn{6}{|l|}{ Laboratory data } \\
\hline Hemoglobin $(\mathrm{g} / \mathrm{dL})$ & $13.5 \pm 1.9$ & $12.9 \pm 2.2$ & 0.02 & 0.13 & 0.02 \\
\hline B-type natriuretic peptide $(\mathrm{pg} / \mathrm{ml})$ & $57.7(19.2-166.7)$ & $257.6(98.9-526.4)$ & $<0.01$ & -0.28 & $<0.01$ \\
\hline Creatinine $(\mathrm{mg} / \mathrm{dL})$ & $0.92 \pm 0.37$ & $1.26 \pm 0.94$ & $<0.01$ & -0.22 & $<0.01$ \\
\hline eGFR $\left(\mathrm{mL} / \mathrm{min} / 1.73 \mathrm{~cm}^{2}\right)$ & $62.4 \pm 17.2$ & $49.1 \pm 16.5$ & $<0.01$ & 0.31 & $<0.01$ \\
\hline Sodium $(\mathrm{mEq} / \mathrm{L})$ & $139.7 \pm 2.7$ & $139.2 \pm 3.5$ & 0.14 & 0.10 & 0.06 \\
\hline $\begin{array}{l}\text { Urinary protein creatinine ratio }\left(\mathrm{g} / \mathrm{g}{ }^{*} \mathrm{Creati}-\right. \\
\text { nine) }\end{array}$ & $0(0-0.06)$ & $0(0-0.25)$ & 0.07 & -0.14 & 0.01 \\
\hline $\begin{array}{l}\text { Urinary albumin creatinine ratio }(\mathrm{mg} / \mathrm{g} \\
\left.{ }^{*} \text { Creatinine }\right)\end{array}$ & $11.5(5.0-35.8)$ & $25.0(9-125.5)$ & 0.04 & -0.21 & $<0.01$ \\
\hline Urinary $\beta 2$ micro globulin $(\mu \mathrm{g} / \mathrm{mL})$ & $0.11(0.06-0.20)$ & $0.12(0.05-0.40)$ & 0.99 & -0.08 & 0.18 \\
\hline Urinary NAG (U/L) & $4.5(2.4-8.7)$ & $6.6(3.1-14.6)$ & 0.02 & -0.16 & $<0.01$ \\
\hline \multicolumn{6}{|l|}{ Echocardiography } \\
\hline LVEF (\%) & $56.1 \pm 15.4$ & $49.3 \pm 16.5$ & $<0.01$ & 0.23 & $<0.01$ \\
\hline Left atrial volume index $\left(\mathrm{mL} / \mathrm{m}^{2}\right)$ & $48.0 \pm 27.7$ & $64.4 \pm 35.2$ & $<0.01$ & -0.20 & $<0.01$ \\
\hline LVOT-VTI $(\mathrm{cm})$ & $18.2 \pm 4.8$ & $15.6 \pm 5.3$ & $<0.01$ & 0.28 & $<0.01$ \\
\hline Mitral valve E/e' & $12.4 \pm 8.0$ & $15.7 \pm 8.8$ & $<0.01$ & -0.22 & $<0.01$ \\
\hline RA end systolic area $\left(\mathrm{cm}^{2}\right)$ & $17.2 \pm 6.3$ & $21.0 \pm 7.2$ & $<0.01$ & -0.25 & $<0.01$ \\
\hline RV area diastole $\left(\mathrm{cm}^{2}\right)$ & $19.6 \pm 7.0$ & $23.2 \pm 9.3$ & 0.04 & -0.26 & $<0.01$ \\
\hline $\mathrm{RV}$ area systole $\left(\mathrm{cm}^{2}\right)$ & $12.0 \pm 5.5$ & $16.4 \pm 8.7$ & $<0.01$ & -0.30 & $<0.01$ \\
\hline RV fractional area change (\%) & $40.0 \pm 10.9$ & $32.8 \pm 12.5$ & $<0.01$ & 0.32 & $<0.01$ \\
\hline Inferior vena cava diameter $(\mathrm{mm})$ & $15.1 \pm 4.0$ & $16.2 \pm 4.8$ & 0.07 & -0.07 & 0.22 \\
\hline TRPG $(\mathrm{mmHg})$ & $24.8 \pm 12.7$ & $29.5 \pm 16.5$ & 0.02 & -0.23 & $<0.01$ \\
\hline \begin{tabular}{|l|} 
Right-heart catherization \\
\end{tabular} & $\mathrm{n}=110$ & $\mathrm{n}=56$ & & & \\
\hline PAP mean $(\mathrm{mmHg})$ & $23.8 \pm 9.8$ & $29.5 \pm 12.3$ & $<0.01$ & -0.26 & $<0.01$ \\
\hline PAP systolic $(\mathrm{mmHg})$ & $35.5 \pm 14.9$ & $42.6 \pm 20.5$ & $<0.01$ & -0.24 & $<0.01$ \\
\hline PAP diastolic (mmHg) & $14.9 \pm 7.2$ & $19.9 \pm 8.6$ & $<0.01$ & -0.29 & $<0.01$ \\
\hline Right atrial pressure mean $(\mathrm{mmHg})$ & $6.7 \pm 3.7$ & $8.5 \pm 3.6$ & $<0.01$ & -0.17 & 0.03 \\
\hline PAWP mean $(\mathrm{mmHg})$ & $13.5 \pm 6.4$ & $17.7 \pm 8.8$ & $<0.01$ & -0.18 & 0.02 \\
\hline Cardiac output (L/min) & $4.22 \pm 1.19$ & $3.98 \pm 1.02$ & 0.16 & 0.13 & 0.09 \\
\hline Cardiac index $\left(\mathrm{L} / \mathrm{min} / \mathrm{m}^{2}\right)$ & $2.65 \pm 0.64$ & $2.36 \pm 0.69$ & 0.04 & 0.27 & $<0.01$ \\
\hline \multicolumn{6}{|l|}{ Medication } \\
\hline RAS inhibitors (n, \%) & $180(77.9)$ & $77(74.8)$ & 0.57 & -0.18 & 0.53 \\
\hline$\beta$-Blockers (n, \%) & $128(55.4)$ & $76(73.8)$ & $<0.01$ & 0.31 & $<0.01$ \\
\hline Diuretics (n, \%) & $94(40.7)$ & $77(74.8)$ & $<0.01$ & 0.32 & $<0.01$ \\
\hline Inotropic agents (n, \%) & $14(6.1)$ & $16(15.5)$ & $<0.01$ & 0.28 & $<0.01$ \\
\hline
\end{tabular}

Table 1. Comparisons of patient characteristics between interlobular renal artery VTI groups. VTI velocity time integral, NYHA New York Heart Association, eGFR estimated glomerular filtration rate, NAG N-acetyl$\beta$-D-glucosaminidase, $L V E F$ left ventricular (LV) ejection fraction, $L V O T$ LV outflow tract, $R A$ right atrial, $R V$ right ventriclar, TRPG tricuspid regurgitation pressure gradient, $P A P$ pulmonary artery pressure, $P A W P$ pulmonary artery wedge pressure, $R A S$ renin-angiotensin system. 


\begin{tabular}{|c|c|c|c|c|c|}
\hline & \multicolumn{3}{|l|}{ IRVF pattern group } & \multicolumn{2}{|c|}{$\begin{array}{l}\text { Correlation with monophasic } \\
\text { pattern }\end{array}$} \\
\hline & Non-monophasic $(n=305)$ & Monophasic $(n=36)$ & P value & Correlation coefficient & P value \\
\hline \multicolumn{6}{|l|}{ Demographics } \\
\hline Age (years) & $65.9 \pm 12.9$ & $72.0 \pm 11.8$ & $<0.01$ & 0.05 & $<0.01$ \\
\hline Male sex $(\mathrm{n}, \%)$ & $196(64.3)$ & $17(47.2)$ & 0.07 & -0.70 & 0.06 \\
\hline Body mass index $\left(\mathrm{kg} / \mathrm{m}^{2}\right)$ & $23.9 \pm 4.7$ & $22.4 \pm 3.6$ & 0.05 & -0.09 & 0.05 \\
\hline Systolic blood pressure $(\mathrm{mmHg})$ & $119.0 \pm 17.2$ & $117.1 \pm 19.3$ & 0.55 & -0.01 & 0.54 \\
\hline Diastolic blood pressure $(\mathrm{mmHg})$ & $69.6 \pm 12.3$ & $66.7 \pm 14.2$ & 0.20 & -0.02 & 0.19 \\
\hline Heart rate (bpm) & $71.0 \pm 13.8$ & $67.8 \pm 14.7$ & 0.20 & -0.02 & 0.19 \\
\hline NYHA class III or IV (n, \%) & $21(6.9)$ & $2(5.6)$ & 1.00 & -0.23 & 0.76 \\
\hline \multicolumn{6}{|l|}{ Comorbidities } \\
\hline Atrial fibrillation $(\mathrm{n}, \%)$ & $104(34.1)$ & $21(58.3)$ & $<0.01$ & 0.99 & $<0.01$ \\
\hline Hypertension (n, \%) & $183(60.0)$ & $24(66.7)$ & 0.48 & 0.29 & 0.44 \\
\hline Dyslipidemia $(\mathrm{n}, \%)$ & $217(71.1)$ & $23(63.9)$ & 0.44 & -0.33 & 0.37 \\
\hline Diabetes mellitus (n, \%) & $101(33.1)$ & $11(30.6)$ & 0.85 & -0.19 & 0.76 \\
\hline Chronic kidney disease (n, \%) & $154(50.5)$ & $24(66.7)$ & 0.08 & 0.67 & 0.07 \\
\hline Anemia $(\mathrm{n}, \%)$ & $130(42.6)$ & $20(55.6)$ & 0.16 & 0.52 & 0.14 \\
\hline \multicolumn{6}{|l|}{ Laboratory data } \\
\hline Hemoglobin $(\mathrm{g} / \mathrm{dL})$ & $13.4 \pm 2.0$ & $12.7 \pm 2.1$ & 0.06 & -0.17 & 0.06 \\
\hline B-type natriuretic peptide $(\mathrm{pg} / \mathrm{ml})$ & $86.4(23.5-251.8)$ & $218.9(142.9-547.9)$ & $<0.01$ & 0.01 & 0.01 \\
\hline Creatinine (mg/dL) & $1.06 \pm 0.78$ & $1.04 \pm 0.39$ & 0.87 & -0.04 & 0.87 \\
\hline eGFR $\left(\mathrm{mL} / \mathrm{min} / 1.73 \mathrm{~cm}^{2}\right)$ & $58.8 \pm 19.3$ & $52.1 \pm 15.0$ & 0.04 & -0.02 & 0.04 \\
\hline Sodium $(\mathrm{mEq} / \mathrm{L})$ & $139.5 \pm 2.9$ & $139.9 \pm 3.6$ & 0.50 & 0.04 & 0.50 \\
\hline $\begin{array}{l}\text { Urinary protein creatinine ratio (g/g } \\
{ }^{*} \text { Creatinine) }\end{array}$ & $0(0-0.09)$ & $0(0-0.203)$ & 0.54 & -0.17 & 0.69 \\
\hline $\begin{array}{l}\text { Urinary albumin creatinine ratio } \\
\left(\mathrm{mg} / \mathrm{g}{ }^{*} \text { Creatinine }\right)\end{array}$ & $14.5(6-42.8)$ & $18.5(8-87.5)$ & 0.67 & 0.01 & 0.75 \\
\hline Urinary $\beta 2$ micro globulin $(\mu \mathrm{g} / \mathrm{mL})$ & $011(0.05-0.24)$ & $0.12(0.05-0.28)$ & 0.49 & -0.02 & 0.69 \\
\hline Urinary NAG (U/L) & $4.8(2.4-9.7)$ & $6.2(3.8-15.0)$ & 0.35 & 0.01 & 0.29 \\
\hline \multicolumn{6}{|l|}{ Echocardiography } \\
\hline LVEF (\%) & $54.5 \pm 15.3$ & $49.2 \pm 19.5$ & 0.06 & -0.02 & 0.06 \\
\hline Left atrial volume index $\left(\mathrm{mL} / \mathrm{m}^{2}\right)$ & $51.0 \pm 29.4$ & $74.7 \pm 38.0$ & $<0.01$ & 0.02 & $<0.01$ \\
\hline LVOT-VTI (cm) & $17.3 \pm 5.0$ & $17.3 \pm 6.0$ & 0.99 & 0.01 & 0.99 \\
\hline Mitral valve E/e' & $12.8 \pm 7.8$ & $18.8 \pm 10.9$ & $<0.01$ & 0.06 & $<0.01$ \\
\hline RA end-systolic area $\left(\mathrm{cm}^{2}\right)$ & $17.8 \pm 6.7$ & $23.1 \pm 7.3$ & $<0.01$ & 0.10 & $<0.01$ \\
\hline $\mathrm{RV}$ area diastole $\left(\mathrm{cm}^{2}\right)$ & $20.6 \pm 7.7$ & $20.9 \pm 8.8$ & 0.88 & 0.01 & 0.87 \\
\hline $\mathrm{RV}$ area systole $\left(\mathrm{cm}^{2}\right)$ & $13.1 \pm 6.6$ & $14.0 \pm 8.4$ & 0.63 & 0.02 & 0.63 \\
\hline RV fractional area change (\%) & $38.0 \pm 11.5$ & $35.1 \pm 13.9$ & 0.34 & -0.02 & 0.34 \\
\hline Inferior vena cava diameter $(\mathrm{mm})$ & $15.3 \pm 4.3$ & $17.0 \pm 4.2$ & 0.03 & 0.09 & 0.03 \\
\hline TRPG (mmHg) & $25.4 \pm 12.6$ & $34.3 \pm 21.6$ & $<0.01$ & 0.03 & $<0.01$ \\
\hline Right-heart catherization & $\mathrm{n}=139$ & $\mathrm{n}=27$ & & & \\
\hline PAP mean $(\mathrm{mmHg})$ & $25.3 \pm 10.9$ & $29.2 \pm 11.6$ & 0.11 & 0.03 & 0.10 \\
\hline PAP systolic $(\mathrm{mmHg})$ & $37.4 \pm 16.6$ & $41.8 \pm 20.8$ & 0.23 & 0.01 & 0.24 \\
\hline PAP diastolic (mmHg) & $16.3 \pm 8.0$ & $19.0 \pm 8.0$ & 0.31 & 0.04 & 0.12 \\
\hline Right atrial pressure mean $(\mathrm{mmHg})$ & $7.2 \pm 3.9$ & $9.0 \pm 2.7$ & 0.03 & 0.10 & 0.02 \\
\hline PAWP mean $(\mathrm{mmHg})$ & $14.7 \pm 7.7$ & $16.6 \pm 6.0$ & 0.21 & 0.03 & 0.21 \\
\hline Cardiac output (L/min) & $4.19 \pm 1.20$ & $4.07 \pm 1.15$ & 0.65 & -0.08 & 0.65 \\
\hline Cardiac index $\left(\mathrm{L} / \mathrm{min} / \mathrm{m}^{2}\right)$ & $2.59 \pm 0.68$ & $2.66 \pm 0.59$ & 0.60 & 0.16 & 0.59 \\
\hline \multicolumn{6}{|l|}{ Medication } \\
\hline RAS inhibitors (n, \%) & $234(76.7)$ & $30(83.3)$ & 0.53 & 0.42 & 0.37 \\
\hline$\beta$-Blockers (n, \%) & $183(60.0)$ & $25(69.4)$ & 0.37 & 0.42 & 0.27 \\
\hline Diuretics (n, \%) & $147(48.2)$ & $28(77.8)$ & $<0.01$ & 0.87 & $<0.01$ \\
\hline Inotropic agents $(\mathrm{n}, \%)$ & $29(9.5)$ & $1(2.8)$ & 0.34 & 0.27 & 0.21 \\
\hline
\end{tabular}

Table 2. Comparisons of patient characteristics among IRVF patterns. IRVF intrarenal venous flow, NYHA New York Heart Association, Egfr estimated glomerular filtration rate, $N A G N$-acetyl- $\beta$-D-glucosaminidase, $L V E F$ left ventricular (LV) ejection fraction, LVOT LV outflow tract, VTI velocity time integral, $R A$ right atrial, $R V$ right ventricular, TRPG tricuspid regurgitation pressure gradient, $P A P$ pulmonary artery pressure, $P A W P$ pulmonary artery wedge pressure, $R A S$ renin-angiotensin system. 


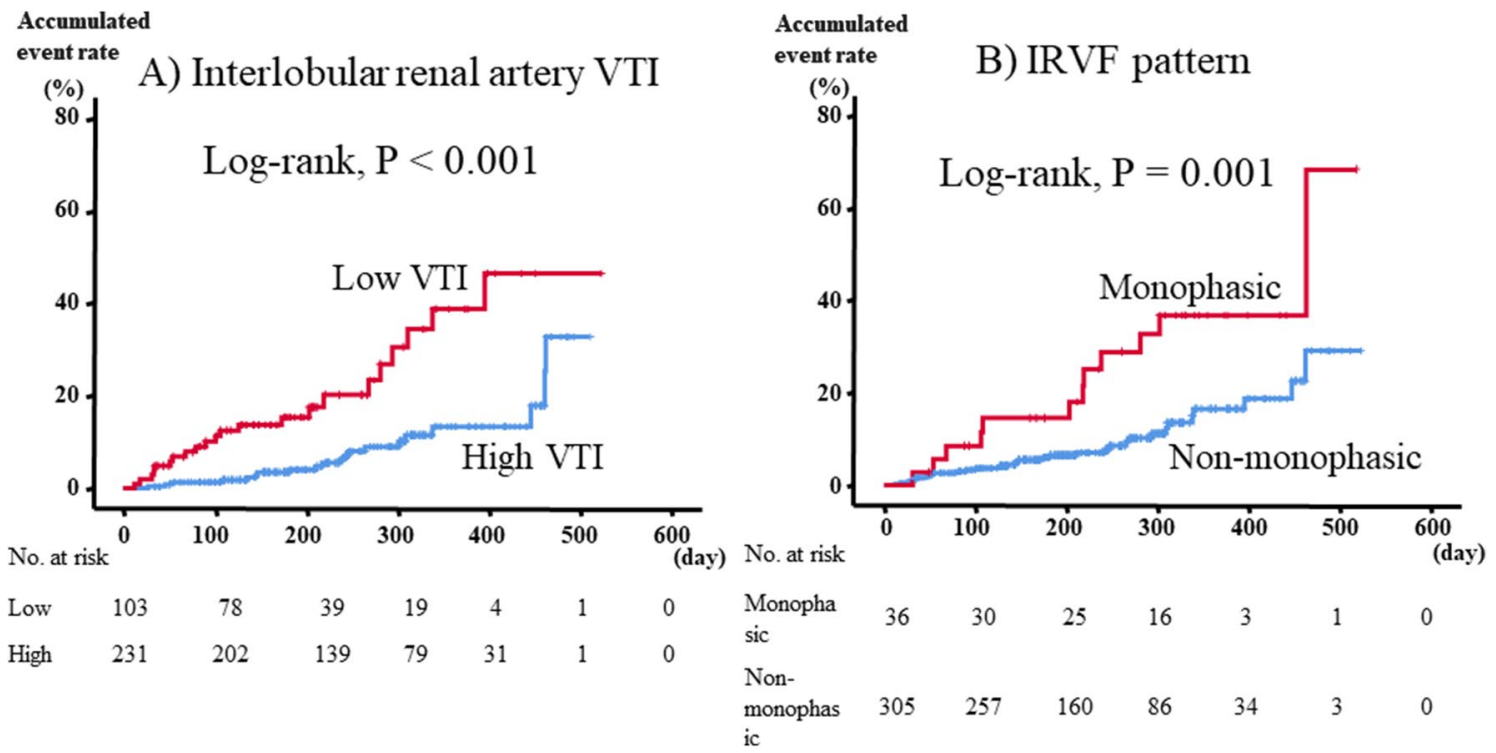

Figure 1. Kaplan-Meier analysis for cardiac events. VTI velocity time integral, IRVF intrarenal venous flow.

\section{Cardiac events stratified by combination of VTI and IRVF pattern}

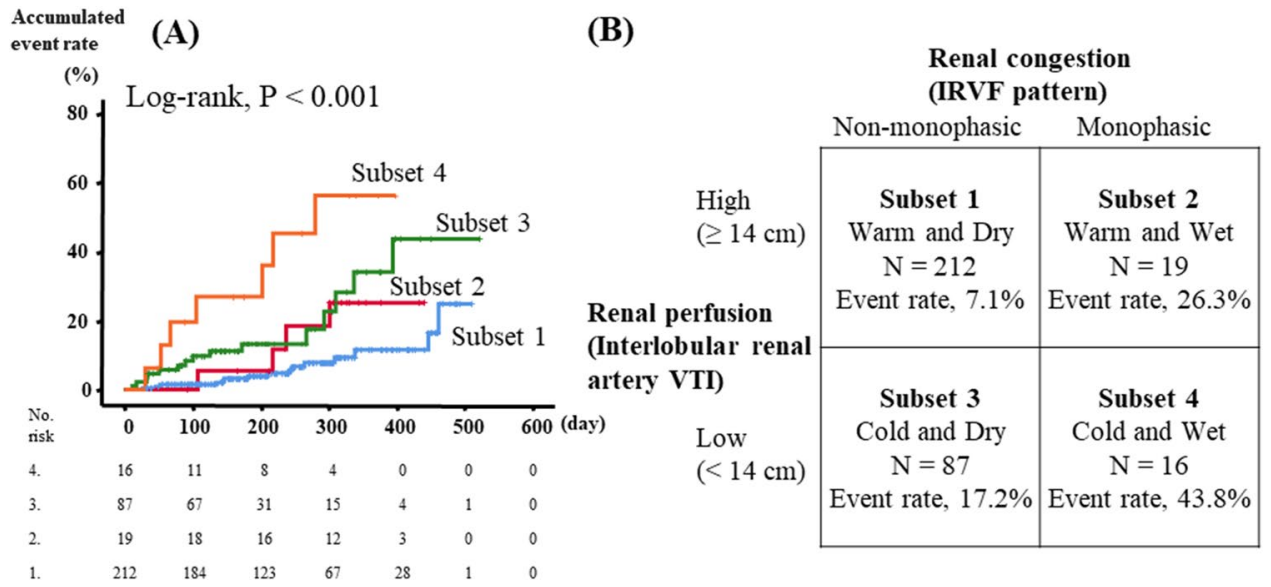

Figure 2. Cardiac events stratified by combination of VTI and IRVF pattern. VTI velocity time integral, IRVF intrarenal venous flow.

the nephrons and the adenosine triphosphate depletion activates epithelial cellular injury and death via necrosis or apoptosis, or both, which ultimately leads to endothelial injury, activation of inflammatory processes, and renal dysfunction ${ }^{35}$. Subsequently, afferent glomerular arterial vasoconstriction occurs and secretes renin, which further activates the renin-angiotensin system and results in efferent glomerular arterial vasoconstriction ${ }^{15}$.

The current study reported the associations between impaired intrarenal perfusion and increased intrarenal congestion, as well as both cardiac function and prognosis. The combination of interlobar renal VTI and IRVF patterns might be a useful tool for evaluating renal hemodynamic subsets such as the Nohria-Stevenson profiles, and may be a therapeutic indicator for managing organ perfusion and congestion.

Study limitations are as follows: first, the number of patients was relatively small and the follow-up period was comparatively short, because the study was carried out in a single center. We could not fully adjust confounding factors in the Cox proportional hazard analysis. Second, although HF patients with renal artery stenosis, dialysis or renal atrophy were excluded, we were unable to completely exclude the presence of subclinical renal diseases. Third, since we conducted the present study using variables measured only during hospitalization, changes in variables (e.g. interlobar renal VTI, IRVF patterns) after discharge were not examined. Fourth, since attending physician decided performing RHC, there might be potential selection bias. Therefore, the present results should be viewed as preliminary, and further studies with a larger population and longer follow up period are needed. 


\begin{tabular}{|c|c|c|c|}
\hline Cardiac event ( 42 events/334 patients) & HR & 95\% CI & P value \\
\hline \multicolumn{4}{|l|}{ VTI group } \\
\hline VTI (low vs. high) & 3.65 & $1.97-6.75$ & $<0.01$ \\
\hline VTI (low vs. high)* & 3.06 & $1.62-5.78$ & $<0.01$ \\
\hline \multicolumn{4}{|l|}{ IRVF pattern group } \\
\hline IRVF pattern (monophasic vs. non-monophasic) & 2.86 & $1.46-5.59$ & $<0.01$ \\
\hline IRVF pattern (monophasic vs. non-monophasic)* & 2.25 & $1.11-4.55$ & 0.02 \\
\hline \multicolumn{4}{|l|}{ Combination of VTI and IRVF pattern } \\
\hline Subset 1 (high VTI and non-monophasic IRVF) & Reference & - & - \\
\hline Subset 2 (high VTI and monophasic IRVF) & 2.92 & $1.06-8.07$ & 0.04 \\
\hline Subset 2 (high VTI and monophasic IRVF)* & 2.09 & $0.74-5.93$ & 0.16 \\
\hline Subset 3 (low VTI and non-monophasic IRVF) & 3.57 & $1.73-7.38$ & $<0.01$ \\
\hline Subset 3 (low VTI and non-monophasic IRVF)* & 2.92 & $1.39-6.14$ & $<0.01$ \\
\hline Subset 4 (low VTI and monophasic IRVF) & 8.36 & $3.37-20.75$ & $<0.01$ \\
\hline Subset 4 (low VTI and monophasic IRVF)* & 6.47 & $2.50-16.77$ & $<0.01$ \\
\hline
\end{tabular}

Table 3. Cox proportional hazard model of cardiac events (factors in parameters of renal ultrasonography). $H R$ hazard ratio, CI confidence interval, VTI velocity time integral, IRVF intrarenal venous flow. *Adjusted: adjusted for age and sex.

\section{Methods}

Subjects and study protocol. This was a prospective observational study of a total of 380 decompensated HF patients, who had undergone abdominal ultrasonography and were discharged from Fukushima Medical University Hospital between April 2018 and March 2019. The diagnosis of HF was defined by cardiologists based on the Framingham criteria, characterized by typical symptoms (e.g. breathlessness and fatigue) and accompanied signs (e.g. elevated jugular venous pressure, pulmonary crackles and peripheral edema). Cardiologists decided needs of hospitalization due to decompensated and/or worsening heart failure in all cases, when managements were necessary such as intravenous agents, respiratory care, dialysis, mechanical support, and etc $^{1-3}$. Blood samples, abdominal ultrasonography and echocardiography were obtained at hospital discharge. Patients with poor quality of images from abdominal ultrasonography $(n=4)$, renal artery stenosis $(n=28)$, and/ or end-stage renal disease receiving dialysis or renal atrophy $(n=7)$ were excluded. Of these 341 patients, RHC was partly performed in 166, among whom, in order to assess the interlobar renal artery VTI that could predict preserved cardiac index $\left(\geq 2.2 \mathrm{~L} / \mathrm{min} / \mathrm{m}^{2}\right)$, we measured the area under the curve of the receiver operating curve. A cut-off value with interlobar renal artery VTI of $\geq 14.0 \mathrm{~cm}$ predicted cardiac index $\left(\geq 2.2 \mathrm{~L} / \mathrm{min} / \mathrm{m}^{2}\right)$ with an area under the curve of 0.616 (95\% confidence interval [CI] 0.508-0.696), with a sensitivity of 0.704 and specificity of 0.600 . Of these 341 patients, 7 patients were excluded in VTI analysis due to unclear imaging. Finally, these patients were divided into groups based on A) interlobar renal artery VTI: high VTI group (VTI $\geq 14.0 \mathrm{~cm}$, $\mathrm{n}=231$ ) or low VTI group (VTI $<14.0 \mathrm{~cm}, \mathrm{n}=103)$ and B) IRVF pattern: monophasic group $(\mathrm{n}=36)$ or nonmonophasic group $(\mathrm{n}=305)$.

First, we compared the clinical features and results from laboratory tests, echocardiography and RHC between the groups. In addition, we performed a correlation analysis of associations between levels of both of interlobar renal artery VTI or IRVF patterns, and parameters of laboratory tests, echocardiography, and RHC. Second, the patients were followed up until December 2019 for cardiac events as composites of cardiac death or unplanned re-hospitalization due to decompensated HF. For patients that experienced two or more events, only the first event was included in the analysis. Since these patients visited patient's referring hospital monthly or bi-monthly, status and dates of death were obtained from the patient's medical records. If these data were unavailable, status was ascertained by a telephone call to the patient's referring hospital physician. This study complied with the Declaration of Helsinki and the STROBE (Strengthening the Reporting of Observational Studies in Epidemiology) statement ${ }^{36,37}$. The study protocol was approved by the Ethics Committee of Fukushima Medical University. All patients gave written informed consent. Informed consent from next of kin or legally authorized representatives is provided.

IRD analysis, interlobuar renal artery VTI and IRVF pattern. Abdominal ultrasonography was performed by two experienced sonographers, who were blinded to all clinical data before discharge using an ultrasound system (Aplio i800, Canon Medical Systems, Tochigi, Japan) equipped with a wideband convex i8CX1 multifrequency probe (central frequency 4.0 MHz, range 1.8-6.4 MHz). Patients fasted for at least $12 \mathrm{~h}$ before the examination. IRD was recorded in the right kidney with the patient in the left lateral decubitus position. Color Doppler images were used to determine interlobar vessels. The velocity range of the color Doppler was set to approximately $12-16 \mathrm{~cm} / \mathrm{s}$ in order to determine interlobar vessels, and the sample volume was set based on the color Doppler signals derived from interlobar vessels ${ }^{17}$. Pulsed Doppler waveforms of the interlobar arteries and veins were recorded simultaneously. As shown in Fig. 3, we examined (a) VTI as a marker of intrarenal hypoperfusion, and (b) IRVF patterns as a marker of intrarenal congestion. The spectral Doppler renal blood flow velocities were recorded, and the VTI was measured as the area under the outermost portion of the 
A) Intrarenal artery Doppler ultrasonography: renal perfusion

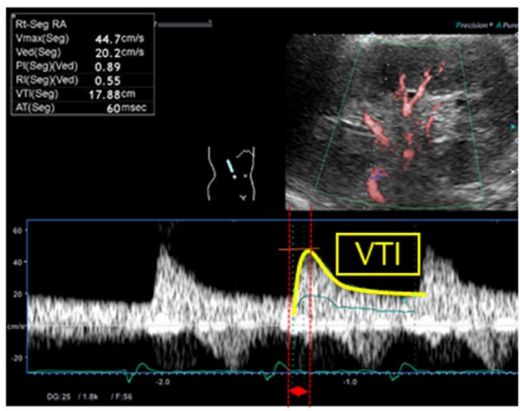

Velocity time integral (VTI)

B) Intrarenal venous flow (IRVF) patterns: renal congestion Artery flow

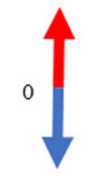

Vein flow

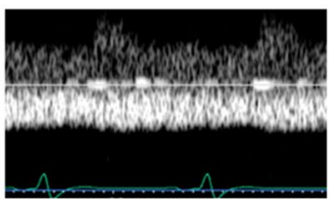

Continuous

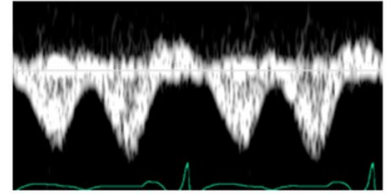

Biphasic

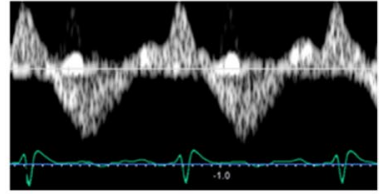

Monophasic

Figure 3. Abdominal ultrasonography.

spectral velocity envelope ${ }^{38}$. Doppler waveforms of IRVF were divided into three flow patterns: continuous, biphasic discontinuous, and monophasic discontinuous (Fig. 3) ${ }^{17,39}$. IRVF patterns are reported to be altered by increases in RAP and are affected by both CVP and reduced renal parenchymal compliance related to intrarenal congestion ${ }^{17}$. It has been reported that monophasic IRVF pattern is especially associated with increased RAP ${ }^{17}$; thus, we focused on monophasic or non-monophasic (continuous-biphasic) IRVF patterns in the present study.

Echocardiography. Patients underwent echocardiography performed by experienced echocardiographers using standard techniques as previously reported ${ }^{40}$. The echocardiographic parameters included LVEF, left atrial volume, LVOT-VTI, mitral valve E/e', right atrium area, RV-FAC, inferior vena cava diameter, and TRPG. All measurements were performed using ultrasound systems (ACUSON Sequoia, Siemens Medical Solutions USA, Inc., Mountain View, CA, USA) ${ }^{40}$.

RHC and hemodynamic measurements. Of the 341 patients, RHC was partly performed based on remedial judgment of the attending physician in 166 patients. RHC was performed with in three days of abdominal ultrasonography, with the patients in a stable condition without changes in medications including doses, similar to setting of abdominal ultrasonography. All RHC was performed as previously reported ${ }^{41}$.

Statistical analysis. Normally distributed data are expressed as mean \pm standard deviation. Non-normally distributed data are presented as median (interquartile range). The categorical variables are expressed as numbers (percentages), and the chi-square test was performed for its comparison. For the comparisons of parametric and non-parametric variables, Student's t test and the Mann-Whitney U test were used, respectively. Associations between VTI and the parameters of laboratory data, echocardiography or RHC, were examined using Pearson's correlation analysis for parametric variables, Spearman's correlation analysis for non-parametric variables. Logistic regression analysis to determine the categorical variables associated with the interlobar renal artery VTI. Logistic regression analysis was also performed to determine the associations between monophasic IRVF pattern and other variables. Kaplan-Meier analysis was used with a log-rank test to assess cardiac event rates. These curves helped in identifying non-proportionality patterns in hazard function such as convergence (difference in risk between the groups decreases with time), divergence, or crossing of the curves. In addition, proportional hazard assumptions were confirmed by log-log analysis. We assessed IRVF patterns and interlobar renal artery VTI levels as predictors for post-discharge cardiac events using the univariate Cox proportional hazard analysis with only age and sex adjusted. Because of small number of events and sample size, as well as the presence of multicollinearity, we did not perform multivariate Cox proportional hazard analyses. In addition, we further classified patients into four subsets in accordance with Nohria-Stevenson clinical profiles: subset 1, high VTI and non-monophasic IRVF patterns (warm-dry); subset 2, high VTI and a monophasic IRVF pattern (warm-wet); subset 3, low VTI and non-monophasic patterns (cold-dry); and subset 4, low VTI and a monophasic IRVF pattern (cold-wet). The predictive value of classification was assessed using the Kaplan-Meier analysis and the Cox proportional hazard analysis. A value of $\mathrm{P}<0.05$ was considered statistically significant for all comparisons. These analyses were performed using SPSS ver. 26.0 (IBM, Armonk, NY, USA). 


\section{Data availability}

The data that support the findings of this study are available from the corresponding author upon reasonable request.

Received: 20 September 2020; Accepted: 8 December 2020

Published online: 17 December 2020

\section{References}

1. Tsutsui, H. et al. JCS 2017/JHFS 2017 guideline on diagnosis and treatment of acute and chronic heart failure-digest version. Circ. J. 83, 2084-2184. https://doi.org/10.1253/circj.CJ-19-0342 (2019).

2. Ponikowski, P. et al. ESC Guidelines for the diagnosis and treatment of acute and chronic heart failure: The Task Force for the diagnosis and treatment of acute and chronic heart failure of the European Society of Cardiology (ESC). Developed with the special contribution of the Heart Failure Association (HFA) of the ESC. Eur. J. Heart Fail. 18, 891-975. https://doi.org/10.1002/ejhf.592 (2016).

3. Yancy, C. W. et al. 2013 ACCF/AHA guideline for the management of heart failure: Executive summary: A report of the American College of Cardiology Foundation/American Heart Association Task Force on practice guidelines. Circulation 128, 1810-1852. https://doi.org/10.1161/CIR.0b013e31829e8807 (2013).

4. Sharma, K. \& Kass, D. A. Heart failure with preserved ejection fraction: Mechanisms, clinical features, and therapies. Circ. Res. 115, 79-96. https://doi.org/10.1161/CIRCRESAHA.115.302922 (2014).

5. Verbrugge, F. H. et al. Abdominal contributions to cardiorenal dysfunction in congestive heart failure. J. Am. Coll. Cardiol. 62, 485-495. https://doi.org/10.1016/j.jacc.2013.04.070 (2013).

6. Yoshihisa, A. et al. Plasma volume status predicts prognosis in patients with acute heart failure syndromes. Eur. Heart J. Acute Cardiovasc. Care 7, 330-338. https://doi.org/10.1177/2048872617690889 (2018).

7. Yoshihisa, A. et al. Liver fibrosis score predicts mortality in heart failure patients with preserved ejection fraction. ESC Heart Fail. 5, 262-270. https://doi.org/10.1002/ehf2.12222 (2018).

8. Sato, Y. et al. Liver stiffness assessed by Fibrosis-4 index predicts mortality in patients with heart failure. Open Heart 4, e000598. https://doi.org/10.1136/openhrt-2017-000598 (2017).

9. Yoshihisa, A. et al. Associations of acid suppressive therapy with cardiac mortality in heart failure patients. J. Am. Heart Assoc. https://doi.org/10.1161/JAHA.116.005110 (2017).

10. Damman, K. et al. Increased central venous pressure is associated with impaired renal function and mortality in a broad spectrum of patients with cardiovascular disease. J. Am. Coll. Cardiol. 53, 582-588. https://doi.org/10.1016/j.jacc.2008.08.080 (2009).

11. Mullens, W. et al. Importance of venous congestion for worsening of renal function in advanced decompensated heart failure. $J$. Am. Coll. Cardiol. 53, 589-596. https://doi.org/10.1016/j.jacc.2008.05.068 (2009).

12. Nohria, A. et al. Clinical assessment identifies hemodynamic profiles that predict outcomes in patients admitted with heart failure. J. Am. Coll. Cardiol. 41, 1797-1804. https://doi.org/10.1016/s0735-1097(03)00309-7 (2003).

13. Hatamizadeh, P. et al. Cardiorenal syndrome: Pathophysiology and potential targets for clinical management. Nat. Rev. Nephrol. 9, 99-111. https://doi.org/10.1038/nrneph.2012.279 (2013).

14. Ronco, C. et al. Cardio-renal syndromes: Report from the consensus conference of the acute dialysis quality initiative. Eur. Heart J. 31, 703-711. https://doi.org/10.1093/eurheartj/ehp507 (2010).

15. Takahama, H. \& Kitakaze, M. Pathophysiology of cardiorenal syndrome in patients with heart failure: Potential therapeutic targets. Am. J. Physiol. Heart Circ. Physiol. 313, H715-H721. https://doi.org/10.1152/ajpheart.00215.2017 (2017).

16. Mullens, W. et al. Evaluation of kidney function throughout the heart failure trajectory-a position statement from the Heart Failure Association of the European Society of Cardiology. Eur. J. Heart Fail. 22, 584-603. https://doi.org/10.1002/ejhf.1697 (2020).

17. Iida, N. et al. Clinical implications of intrarenal hemodynamic evaluation by doppler ultrasonography in heart failure. JACC. Heart Fail. 4, 674-682. https://doi.org/10.1016/j.jchf.2016.03.016 (2016).

18. Nijst, P., Martens, P., Dupont, M., Tang, W. H. W. \& Mullens, W. Intrarenal flow alterations during transition from euvolemia to intravascular volume expansion in heart failure patients. JACC Heart Fail. 5, 672-681. https://doi.org/10.1016/j.jchf.2017.05.006 (2017).

19. Husain-Syed, F. et al. Doppler-derived renal venous stasis index in the prognosis of right heart failure. J. Am. Heart Assoc. 8, e013584. https://doi.org/10.1161/JAHA.119.013584 (2019).

20. de la Espriella-Juan, R. et al. Intrarenal venous flow in cardiorenal syndrome: A shining light into the darkness. ESC Heart Fail. 5, 1173-1175. https://doi.org/10.1002/ehf2.12362 (2018).

21. Beaubien-Souligny, W. et al. Alterations in portal vein flow and intrarenal venous flow are associated with acute kidney injury after cardiac surgery: A prospective observational cohort study. J. Am. Heart Assoc. 7, e009961. https://doi.org/10.1161/JAHA.118.00996 1 (2018).

22. Puzzovivo, A. et al. Renal venous pattern: A new parameter for predicting prognosis in heart failure outpatients. J. Cardiovasc. Dev. Dis. https://doi.org/10.3390/jcdd5040052 (2018).

23. Burnett, J. C. Jr. \& Knox, F. G. Renal interstitial pressure and sodium excretion during renal vein constriction. Am. J. Physiol. 238, F279-282. https://doi.org/10.1152/ajprenal.1980.238.4.F279 (1980).

24. Chiba, H. et al. Renoprotective effects of tolvaptan in hypertensive heart failure rats depend on renal decongestion. Hypertens. Res. 42, 319-328. https://doi.org/10.1038/s41440-018-0169-3 (2019).

25. Yamamoto, M. et al. Prognostic impact of changes in intrarenal venous flow pattern in patients with heart failure. J. Cardiac. Fail. https://doi.org/10.1016/j.cardfail.2020.06.016 (2020).

26. Verbrugge, F. H. et al. The kidney in congestive heart failure: "are natriuresis, sodium, and diuretics really the good, the bad and the ugly?". Eur. J. Heart Fail. 16, 133-142. https://doi.org/10.1002/ejhf.35 (2014).

27. Ross, E. A. Congestive renal failure: The pathophysiology and treatment of renal venous hypertension. J. Cardiac. Fail. 18, 930-938. https://doi.org/10.1016/j.cardfail.2012.10.010 (2012).

28. Colombo, P. C. et al. Peripheral venous congestion causes inflammation, neurohormonal, and endothelial cell activation. Eur. Heart J. 35, 448-454. https://doi.org/10.1093/eurheartj/eht456 (2014).

29. Ammons, W. S., Koyama, S. \& Manning, J. W. Neural and vascular interaction in renin response to graded renal nerve stimulation. Am. J. Physiol. 242, R552-562. https://doi.org/10.1152/ajpregu.1982.242.5.R552 (1982).

30. Firth, J. D., Raine, A. E. \& Ledingham, J. G. Raised venous pressure: A direct cause of renal sodium retention in oedema?. Lancet 1, 1033-1035. https://doi.org/10.1016/s0140-6736(88)91851-x (1988).

31. Kalra, P. R., Anagnostopoulos, C., Bolger, A. P., Coats, A. J. \& Anker, S. D. The regulation and measurement of plasma volume in heart failure. J. Am. Coll. Cardiol. 39, 1901-1908. https://doi.org/10.1016/s0735-1097(02)01903-4 (2002).

32. Mohamed, B. A. et al. Molecular and structural transition mechanisms in long-term volume overload. Eur. J. Heart Fail. 18, 362-371. https://doi.org/10.1002/ejhf.465 (2016). 
33. Testani, J. M., Chen, J., McCauley, B. D., Kimmel, S. E. \& Shannon, R. P. Potential effects of aggressive decongestion during the treatment of decompensated heart failure on renal function and survival. Circulation 122, 265-272. https://doi.org/10.1161/CIRCU LATIONAHA.109.933275 (2010).

34. Bell, P. D., Lapointe, J. Y. \& Peti-Peterdi, J. Macula densa cell signaling. Annu. Rev. Physiol. 65, 481-500. https://doi.org/10.1146/ annurev.physiol.65.050102.085730 (2003).

35. Ronco, C., Bellomo, R. \& Kellum, J. A. Acute kidney injury. Lancet 394, 1949-1964. https://doi.org/10.1016/S0140-6736(19)32563 -2 (2019).

36. Rickham, P. P. Human experimentation. Code of Ethics of the World Medical Association. Declaration of Helsinki. Br. Med. J. 2, 177. https://doi.org/10.1136/bmj.2.5402.177 (1964).

37. von Elm, E. et al. Strengthening the Reporting of Observational Studies in Epidemiology (STROBE) statement: Guidelines for reporting observational studies. BMJ 335, 806-808. https://doi.org/10.1136/bmj.39335.541782.AD (2007).

38. Horita, Y. et al. Renal circulatory effects of acetazolamide in patients with essential hypertension. Am. J. Hypertens. 19, $282-285$. https://doi.org/10.1016/j.amjhyper.2005.09.014 (2006).

39. Tang, W. H. \& Kitai, T. Intrarenal venous flow: A window into the congestive kidney failure phenotype of heart failure?. JACC. Heart Fail. 4, 683-686. https://doi.org/10.1016/j.jchf.2016.05.009 (2016).

40. Yoshihisa, A. et al. Soluble neprilysin does not correlate with prognosis in pulmonary hypertension. ESC Heart Fail. 6, $291-296$. https://doi.org/10.1002/ehf2.12404 (2019).

41. Yoshihisa, A. et al. Liver fibrosis marker, 7S domain of collagen type IV, in patients with pre-capillary pulmonary hypertension. Int. J. Cardiol. 258, 269-274. https://doi.org/10.1016/j.ijcard.2018.01.138 (2018).

\section{Acknowledgements}

The authors thank Ms. Tomiko Miura, Ms. Kumiko Watanabe and Ms. Yumi Yoshihisa for their technical assistance.

\section{Author contributions}

A.Y., K.W., Y.S., S.I., M.M., Y.Y., Y.I., T.Y., T.M., M.O., A.K. and Y.T. contributed to the conception or design of the work. A.Y., K.W., Y.S., S.I., M.M., Y.Y., Y.I., T.Y., T.M., M.O. and A.K. contributed to the acquisition, analysis, or interpretation of data for the work. A.Y., K.W. and Y.T. drafted the manuscript. A.Y., K.W., Y.S., S.I., M.M., Y.Y., Y.I., T.Y., T.M., M.O., A.K. and Y.T. critically revised the manuscript. All gave final approval and agree to be accountable for all aspects of work ensuring integrity and accuracy.

\section{Funding}

This study was supported in part by a Grant-in-Aid for Scientific Research (no. 20K07828) from the Japan Society for the Promotion of Science.

\section{Competing interests}

The authors declare no competing interests.

\section{Additional information}

Correspondence and requests for materials should be addressed to A.Y.

Reprints and permissions information is available at www.nature.com/reprints.

Publisher's note Springer Nature remains neutral with regard to jurisdictional claims in published maps and institutional affiliations.

Open Access This article is licensed under a Creative Commons Attribution 4.0 International License, which permits use, sharing, adaptation, distribution and reproduction in any medium or format, as long as you give appropriate credit to the original author(s) and the source, provide a link to the Creative Commons licence, and indicate if changes were made. The images or other third party material in this article are included in the article's Creative Commons licence, unless indicated otherwise in a credit line to the material. If material is not included in the article's Creative Commons licence and your intended use is not permitted by statutory regulation or exceeds the permitted use, you will need to obtain permission directly from the copyright holder. To view a copy of this licence, visit http://creativecommons.org/licenses/by/4.0/.

(C) The Author(s) 2020 